\title{
Erratum to: Simple scoring system for prediction of mortality in Fournier's gangrene
}

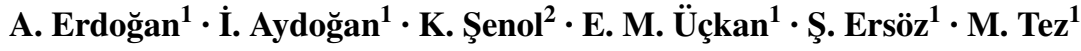

Published online: 11 September 2017

(c) Springer-Verlag GmbH Germany 2017

\section{Erratum to: Eur J Trauma Emerg Surg (2016) 42:513-518 DOI 10.1007/s00068-015-0572-2}

As mentioned in the results, there were 92 patients admitted to the emergency department, but 84 patient's data were eligible for statistical analyses. Therefore 8 patients were excluded from the study. Of the 84 patients, 74 (88\%) were survivors.

In the manuscript, demographic and clinical characteristics of the survivor and non-survivor groups were compared in the Table 1 .

However, in that table, there was an unintended miscalculation of the patients with colostomy in the survivor group. 8 patients who are excluded from the study were mistakenly added to the survivor group with colostomy.

Statistical analyses performed with the correct numbers did not show any significant difference between survivor and non-survivor group of patients with or-without colostomy. Therefore, this miscalculation did not affect the data interpretation and study design.

It would be appropriate to correct the data in the survivor group, patients with or without colostomy as 59 patients and 15 patients.

The correct Table 1 is given below.
The online version of the original article can be found under doi:10.1007/s00068-015-0572-2.

M. Tez

mesuttez@yahoo.com

1 Ankara Numune Education and Research Hospital, Cengizhan cad., 10/3 Bahçelievler, 06500 Ankara, Turkey

2 Bursa State Hospital, Bursa, Turkey 
Table 1 Demographic and clinical characteristics of patients

\begin{tabular}{|c|c|c|c|}
\hline Variables & $\begin{array}{l}\text { Exitus }(n=10)(\text { mean, } \\
\text { standard deviation) }\end{array}$ & $\begin{array}{l}\text { Survivor }(n=74) \text { (mean, } \\
\text { standard deviation) }\end{array}$ & $p$ value \\
\hline Gender female/male & $2 / 8$ & $29 / 45$ & NS \\
\hline Age & $68 \pm 12$ & $54 \pm 14$ & .007 \\
\hline WBC & $12.70 \pm 7.38$ & $14.76 \pm 6.10$ & NS \\
\hline Hemoglobin & $10.15 \pm 1.51$ & $11.88 \pm 2.33$ & .013 \\
\hline Platelet & $259.50 \pm 150.04$ & $325.89 \pm 161.37$ & NS \\
\hline RDW & $17.24 \pm 3.39$ & $14.63 \pm 2.45$ & .004 \\
\hline Fasting blood glucose & $135.10 \pm 41.49$ & $158.94 \pm 98.73$ & NS \\
\hline Urea & $68.00 \pm 33.51$ & $38.43 \pm 30.15$ & .005 \\
\hline Creatinine & $1.20 \pm .93$ & $.93 \pm .60$ & NS \\
\hline ALT & $23.80 \pm 13.35$ & $25.59 \pm 16.49$ & NS \\
\hline AST & $34.20 \pm 23.17$ & $28.21 \pm 17.21$ & NS \\
\hline ALP & $120.10 \pm 88.60$ & $94.48 \pm 42.90$ & NS \\
\hline GGT & $56.10 \pm 68.55$ & $53.75 \pm 40.10$ & NS \\
\hline LDH & $276.10 \pm 125.01$ & $229.55 \pm 122.01$ & NS \\
\hline Total protein & $49.96 \pm 8.55$ & $59.79 \pm 9.99$ & .007 \\
\hline Albumin & $16.89 \pm 3.99$ & $27.05 \pm 8.92$ & .000 \\
\hline Total calcium & $7.39 \pm .61$ & $8.48 \pm 1.05$ & .000 \\
\hline Total bilirubin & $.74 \pm .45$ & $.89 \pm .91$ & NS \\
\hline LOS & $25 \pm 18$ & $27 \pm 24$ & NS \\
\hline Co-morbid disease $( \pm)$ & $10 / 0$ & $38 / 44$ & .001 \\
\hline Presence of sepsis $( \pm)$ & $10 / 0$ & $7 / 75$ & .000 \\
\hline Culture result $( \pm)$ & $5 / 5$ & $46 / 36$ & NS \\
\hline Colostomy $( \pm)$ & $1 / 9$ & $59 / 15$ & NS \\
\hline Surgical procedure & & & NS \\
\hline Debridement & 10 & 68 & \\
\hline Debridement + graft & 0 & 1 & \\
\hline Debridement + flap & 0 & 5 & \\
\hline
\end{tabular}

